\title{
Edge Majoranas on locally flat surfaces: The cone and the Möbius band
}

\author{
A. Quelle and C. Morais Smith \\ Institute for Theoretical Physics, Center for Extreme Matter and Emergent Phenomena, Utrecht University, \\ Leuvenlaan 4, 3584 CE Utrecht, Netherlands \\ T. Kvorning \\ Department of Physics, Stockholm University, AlbaNova University Center, SE-106 91 Stockholm, Sweden \\ T. H. Hansson \\ Department of Physics, Stockholm University, AlbaNova University Center, SE-106 91 Stockholm, Sweden \\ and NORDITA, Roslagstullsbacken 23, SE-106 91 Stockholm, Sweden
}

(Received 21 June 2016; published 21 September 2016)

\begin{abstract}
In this paper, we investigate the edge Majorana modes in the simplest possible $p_{x}+i p_{y}$ superconductor defined on surfaces with different geometries, the annulus, the cylinder, the Möbius band, and a cone (by cone we mean a cone with the tip cut away so it is topologically equivalent to the annulus and cylinder), and with different configurations of magnetic fluxes threading holes in these surfaces. In particular, we shall address two questions: Given that, in the absence of any flux, the ground state on the annulus does not support Majorana modes while the one on the cylinder does, how is it possible that the conical geometry can interpolate smoothly between the two? Given that in finite geometries edge Majorana modes have to come in pairs, how can a $p_{x}+i p_{y}$ state be defined on a Möbius band, which has only one edge? We show that the key to answering these questions is that the ground state depends on the geometry, even though all the surfaces are locally flat. In the case of the truncated cone, there is a nontrivial holonomy, while the nonorientable Möbius band must necessarily support a domain wall.
\end{abstract}

DOI: 10.1103/PhysRevB.94.125137

\section{INTRODUCTION}

Among all the fascinating features that topological states of matter exhibit, excitations with non-Abelian statistics are one of the most intriguing. They were first predicted to occur in quantum Hall systems at filling fraction $v=5 / 2$, $v=7 / 2$ (see Refs. [1,2]) and later at other fractions in higher Landau levels [3]. In addition, they are expected to arise in two-dimensional (2D) spinless $p_{x}+i p_{y}$ superconductors, where the vortices can be shown to obey non-Abelian Ising statistics [4-7]. The anyonic nature of the vortices in such a superconductor originate from the presence of zero-energy Majorana modes in the vortex cores (in what follows, Majorana will always refer to a zero-energy Majorana mode). Such Majorana modes have not yet been observed in 2D systems, but there is experimental evidence pointing to their existence in one-dimensional topological superconductors [8,9].

In 2D superconductors, the presence of such Majorana modes is determined by the amount of flux piercing the superconductor at a vortex: an odd number of superconducting flux quanta (unit flux) gives a Majorana, while an even number does not. Since Majorana modes can only come in pairs, an odd total number of unit flux vortices requires the presence of a Majorana mode at the boundary of the material. To understand this point, it is important to realize that a topological superconductor always has gapless edge modes in the thermodynamic limit, but there is not necessarily an edge mode precisely at zero energy (an edge Majorana) for finite edge length [4]. To know the number of Majorana modes in the presence of an arbitrary number of unit fluxes, one needs to know how many Majorana modes are present in the absence of flux. If the boundary consists of multiple edges, an even number of edge Majoranas might be present in the absence of flux.
To answer this question, one has to realize that the $p$-wave order parameter is not, as in the $s$-wave case, just a scalar field. Rather, it is a vector field, and consequently, it couples to the geometry of the surface $[10,11]$. At first, one would think that only the local curvature of the surface would matter, so that, for example, a cylinder and an annulus would be indistinguishable because they are both locally flat and have the same topology. This is, however, not true, since it is known that in the absence of magnetic flux, the cylinder does support Majorana modes, while the annulus does not [4].

The solution to this puzzle is provided by investigating a cone, where we let the conical angle smoothly interpolate between the annulus and the cylinder. In this paper, we show that there is a crossover between the ground state and the first excited state at a critical opening angle of the cone and that, in general, the ground state supports a current. These effects all depend on the internal geometry of the cone, but it is also interesting to study effects that depend on the topology. An interesting example is provided by a Möbius band.

Since a Möbius band is nonorientable, one would naively think that it cannot possibly support a chiral superconducting state. On the other hand, for a flat or moderately curved strip, such a state should clearly exist locally, which is possible if one allows for line defects between states of different chirality [12]. Here, we will show that there are two physically different minimal choices for the line defect, one where the defect is across the Möbius band, which makes the Möbius band essentially like the disk, and another one for which the defect is along the center line, which makes the geometry similar to a cylinder.

The Möbius band has only one boundary component; hence, the response to external fluxes and the interplay between vortex 
excitations and edge Majoranas differ from those of an annulus or a cylinder. We will show that when the defect line is along the center of the Möbius band, the Majorana at the defect cannot be removed by the addition of a unit flux. The existence of a Majorana at the line defect is only affected by half a unit of flux passing through the hole of the band. These effects can be understood by mapping the Möbius band to a corresponding cylinder. Although the existence of the Majorana is not affected by one unit of flux passing through the hole of the band, the profile of the Majorana wave function across the line defect will be modified.

The outline of the paper is the following: in Sec. II, we review the physics of a $2 \mathrm{D} p$-wave superconductor in the simplest possible setting, namely, that of spinless electrons. We begin by introducing the model, and then, in the framework of a mean-field approximation, we set the stage for the $p$-wave superconductor. Then, in Sec. III, we consider a general cone and explain why the nontrivial holonomy of the Levi-Civita connection gives rise to a spontaneous current and how this affects the Majorana edge modes. Finally, in Secs. IV and V we discuss the Möbius band and how the Majorana modes in this geometry are affected by inserting flux tubes. Our conclusions are presented in Sec. VI.

\section{TWO-DIMENSIONAL $p$-WAVE SUPERCONDUCTOR}

Let us start by reviewing the formalism for describing a spinless 2D $p_{x}+i p_{y}$ superconductor on a general curved surface and by getting the general form of the allowed boundary condition at the edges. This is the simplest model for a $p$-wave superconductor in two dimensions, which allows for a clear presentation of the relevant features.

Since the Pauli principle prevents a local interaction among spinless electrons, the dominating long-wavelength part of any finite-range two-body interaction, which is only a function of the geodesic distance [13], can be written as $V(x, y)=$ $\lambda \nabla^{2} \delta^{2}(x-y)$, where $\nabla^{2}$ is the Laplacian [14].

The most convenient way to write this interaction in a coordinate-invariant form is to introduce an orthonormal frame: a pair of orthonormal unit vectors $\left\{e_{a}^{\mu}\right\}_{a=1,2}$ at every point. By construction, the metric can be expressed as $g^{\mu \nu}=$ $e_{a}^{\mu} e^{a \nu}$, where $e_{a}^{\mu}=e^{a \mu}$ by definition.

Performing a partial integration and using the fact that the square of any fermionic operator vanishes, we can express the interaction in second-quantized form as

$$
\hat{V}=\lambda \int d S\left[\left(\psi^{\dagger} \partial_{-} \psi^{\dagger}\right)\left(\psi \partial_{+} \psi\right)+\left(\psi^{\dagger} \partial_{+} \psi^{\dagger}\right)\left(\psi \partial_{-} \psi\right)\right],
$$

where $d S=d^{2} x \sqrt{\operatorname{det} g_{\mu \nu}}, \partial_{ \pm}=e_{1}^{\mu} \partial_{\mu} \pm i e_{2}^{\mu} \partial_{\mu}$, and $\psi^{\dagger}$ and $\psi$ are, respectively, the (spinless) electron creation and annihilation operators. by

In a mean-field approach, we approximate the pairing term

$$
\hat{V}=\frac{\lambda}{2} \int d S\left[\left(\psi^{\dagger} \partial_{-} \psi^{\dagger}\right) \phi_{+}+\left(\psi^{\dagger} \partial_{+} \psi^{\dagger}\right) \phi_{-}+\text {H.c. }\right],
$$

where the values of $\phi_{ \pm}=\left\langle\psi \partial_{ \pm} \psi\right\rangle$ should, strictly speaking, be determined self-consistently. However, we shall take them as given background fields with the correct topological properties. For the ground state in the $p_{x}+i p_{y}$ case, we will use $\phi \equiv \phi_{+}=$const and $\phi_{-}=0$.

Since $\phi$ is a vector field with charge $2 e$, constant means covariantly constant. To this end, we recall that, with respect to the normalized basis $\left\{e_{a}^{\mu}\right\}_{a=1,2}$, the covariant derivative of a general vector with components $V^{a}$ is

$$
D_{\mu} V^{a}=\left(\delta_{b}^{a} \partial_{\mu}+\omega_{\mu b}^{a}\right) V^{b},
$$

where the connection form is defined by

$$
\omega_{\mu b}^{a}=e_{\nu}^{a} \nabla_{\mu} e_{b}^{v},
$$

with $\nabla_{\mu}$ being the Levi-Civita connection. It can be shown that $\omega_{\mu b}^{a}$ is antisymmetric in $a$ and $b$. Therefore, the covariant derivative of $V_{ \pm}:=V^{1} \pm i V^{2}$ is $D_{\mu} V_{ \pm}=\left(\partial_{\mu} \pm i \omega_{\mu}\right) V_{ \pm}$, where $\omega_{\mu} \equiv \omega_{\mu 2}^{1}$. In the presence of an electromagnetic vector potential, this generalizes to

$$
D_{\mu} \phi=\left(\partial_{\mu}+2 i e A_{\mu}+i \omega_{\mu}\right) \phi,
$$

where $A_{\mu}$ is the electromagnetic gauge potential. Barring any geometric obstructions, this will vanish in the ground state to minimize the kinetic energy of the system.

The mean-field Hamiltonian reads

$$
\hat{H}=\int d S \Psi^{\dagger}\left(\begin{array}{cc}
h_{0} & \phi \partial_{-} \\
\phi^{*} \partial_{+} & -h_{0}^{*}
\end{array}\right) \Psi,
$$

where $\left(\psi, \psi^{\dagger}\right)^{T}$ and the noninteracting part of the Hamiltonian $h_{0}$ may, in general, contain any power of the Laplacian. However, since we are interested only in long-wavelength effects, we truncate it and keep only the lowest order in derivatives; that is, we take $h_{0}$ to be a constant chemical potential [15], $h_{0} \equiv \mu$. The first-quantized Hamiltonian $\mathcal{H}$ is defined by how it acts on a general single quasiparticle state,

$$
|u, v\rangle=\int d S\left(\psi^{\dagger}, \psi\right)(u, v)^{T}|0\rangle,
$$

where $|0\rangle$ is the ground state, whose existence is assumed. Since the number of quasiparticles is conserved, the Schrödinger equation can be rewritten as a partial differential equation for the functions $u, v$ :

$$
i \hbar \frac{\partial}{\partial t}\left(\begin{array}{l}
u \\
v
\end{array}\right)=\mathcal{H}\left(\begin{array}{l}
u \\
v
\end{array}\right)
$$

Here, the first-quantized Hamiltonian reads

$$
\mathcal{H}=\left(\begin{array}{lc}
-\mu & \frac{1}{2 \sqrt{g}}\left\{\sqrt{g} \phi, \partial_{-}\right\} \\
-\frac{1}{2 \sqrt{g}}\left\{\sqrt{g} \phi^{*}, \partial_{+}\right\} & \mu
\end{array}\right),
$$

where $\{\cdot, \cdot\}$ denotes, as usual, the anticommutator. The most general local boundary condition for which this Hamiltonian is self-adjoint reads

$$
\left.\hat{n}^{\mu} e_{\mu}^{a} \sigma_{a}\left(\begin{array}{c}
\phi^{*} u \\
\phi v
\end{array}\right)\right|_{\partial S}=\left.s \Delta\left(\begin{array}{l}
u \\
v
\end{array}\right)\right|_{\partial S},
$$

where $s$ is an arbitrary real number, $\hat{n}^{\mu}$ is the outward directed normal, $\Delta=|\phi|$, and $\partial S$ is the edge of the surface $S$ on which the system is defined.

The Bogoliubov-de Gennes Hamiltonian satisfies

$$
\sigma_{x} \mathcal{H}^{*} \sigma_{x}=\mathcal{H}
$$




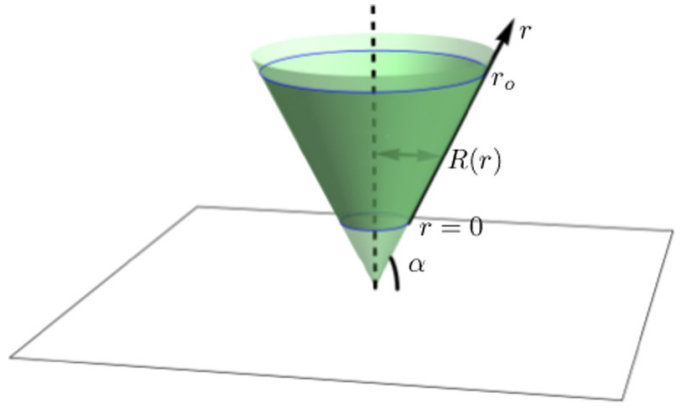

FIG. 1. The embedding given by Eq. (7).

which reflects that in first-quantized language, we formally have doubled the degrees of freedom. This property of the first-quantized Hamiltonian is required for $\psi^{\dagger}$ to be the adjoint of $\psi$ and is thus not a symmetry but a consequence of the second-quantized structure. Therefore, only boundary conditions consistent with (6) are allowed, i.e., $|s|=1$.

\section{EDGE MODES ON THE CONE}

We now focus on a conical surface, defined by the embedding

$$
(r, \theta) \mapsto(R(r) \cos \theta, R(r) \sin \theta, r \sin \alpha),
$$

where $R(r)=R_{i}+r \cos \alpha, \theta \in[0,2 \pi]$, and $r \in\left[0, R_{o}\right]$ (see Fig. 1). For $\alpha=0$, this is an annulus with inner radius $R_{i}$ and outer radius $R_{o}=R\left(r_{o}\right)$, and for $\alpha=\pi / 2$ it is a finite cylinder of radius $R_{i}$ and length $r_{o}$. The parameter $\alpha$ is the angle that the conic surface makes with the $x y$ plane and defines a smooth interpolation between the annulus and the cylinder. The metric in the $(r, \theta)$ coordinates is inherited from the Euclidean one by the embedding (7):

$$
g_{\mu \nu}=\left(\begin{array}{cc}
1 & 0 \\
0 & R(r)^{2}
\end{array}\right)
$$

In this coordinate system, a natural choice of orthonormal frame is the set of unit coordinate vectors,

$$
e_{1}=\hat{r} \equiv \partial_{r}, \quad e_{2}=\hat{\theta} \equiv \frac{1}{R(r)} \partial_{\theta} .
$$

These are the normalized radial and angular vectors along the cone. In terms of the Cartesian-coordinate system on the ambient space in which the cone is embedded, we find

$$
\begin{aligned}
& \hat{r}=(\cos \alpha \cos \theta, \cos \alpha \sin \theta, \sin \alpha), \\
& \hat{\theta}=(-\sin \theta, \cos \theta, 0) .
\end{aligned}
$$

We can use the embedding in Eq. (7) to calculate the connection form, $\omega \equiv \omega_{2}^{1}$ in Eq. (1). It is equal to the Levi-Civita connection for the ambient space projected onto the cone. We obtain

$$
\omega=d \theta \hat{r} \cdot \partial_{\theta} \hat{\theta}+d r \hat{r} \cdot \partial_{r} \hat{\theta}=-\cos \alpha d \theta .
$$

In terms of components this reads $\omega_{\mu}=-R(r) \cos (\alpha) e_{\mu}^{2}$. Here, $e_{\mu}^{2}$ are the components of the one-form dual to the frame field $e_{2}$. For a flat surface, the connection is fully determined by the holonomies $\int_{C} \omega$ around noncontractible curves.
For $n$ units of flux through the hole in the cone and no flux elsewhere, the electromagnetic vector potential reads $A_{\mu}=n(2 e)^{-1} e_{\mu}^{2}$. Due to rotational symmetry, we may take the superconducting order parameter to be

$$
\phi=e^{-i m \theta} \Delta,
$$

where $\Delta$ is some non-negative real number and $m$ is an integer. This implies that

$$
D_{\mu} \phi=i(n-m-\cos \alpha) e_{\mu}^{2} .
$$

This expression can only vanish for the annulus $(\alpha=0)$ and for the cylinder $(\alpha=\pi / 2)$, which means that on a general cone, there is always a nonzero supercurrent present due to the presence of a geometric obstruction. For the annulus the current vanishes for $m=n-1$, while for the cylinder this happens for $m=n$.

A direct calculation yields

$$
\frac{1}{2 \sqrt{g}}\left\{\sqrt{g} \phi, \partial_{-}\right\}=e^{-i m \theta} \Delta\left(\partial_{r}+\frac{i}{R(r)} \partial_{\theta}+\frac{m+\cos \alpha}{2 R(r)}\right) .
$$

Substituting the ansatz

$$
\left(\begin{array}{l}
u \\
v
\end{array}\right)=e^{i l \theta}\left(\begin{array}{c}
e^{-i m \theta / 2} u_{l}(r) \\
e^{i m \theta / 2} v_{l}(r)
\end{array}\right)
$$

into the Hamiltonian (4) for integer $l$ and eliminating $v_{l}$, we obtain

$$
\begin{aligned}
& {\left[\mu^{2}-E^{2}-\Delta^{2}\left(\partial_{r}-\frac{l-\frac{1}{2} \cos \alpha}{R(r)}\right)\right.} \\
& \left.\times\left(\partial_{r}+\frac{l+\frac{1}{2} \cos \alpha}{R(r)}\right)\right] u_{l}(r)=0 .
\end{aligned}
$$

On the inner edge, the boundary condition (5) becomes

$$
v_{l}(0)=s u_{l}(0), \quad s= \pm 1,
$$

or by using the equations of motion,

$$
\left.\left[s(\mu-E)+\Delta\left(\partial_{r}+\frac{l+\frac{1}{2} \cos \alpha}{R(r)}\right)\right] u_{l}(r)\right|_{r=0}=0 .
$$

For $|E|>\mu$, Eq. (14) has plane-wave solutions, so that $\mu$ is the size of the energy gap. From Eq. (14), we also see that for $|E|<\mu$ there are two solutions, one which decays with increasing $r$, and one which increases. Which solution is allowed by the boundary condition (15) depends on the sign of $\mu s$. The decaying solution is only allowed for positive $\mu s$, and the increasing one is only allowed for negative $\mu s$. Since the solutions have to be normalizable, this reflects that a change of the sign of $\mu$ corresponds to a phase boundary between topological and nontopological phases; that is, the phase $\mu s>0$ supports edge modes, while the phase $\mu s<0$ does not [4].

In this analysis, the absolute sign of $\mu$ is of no importance: only the sign relative to the boundary condition, represented by the product $\mu s$, matters. The boundary condition is, as always, fixed by the microscopic physics, i.e., by higherderivative terms. Taking $h_{0}=-\nabla^{2} /(2 M)-\mu$, corresponding to a parabolic band, we will, in the $M \rightarrow \infty$ limit, reobtain the Hamiltonian in Eq. (4), together with the boundary condition 
$s=1$. Thus, for the usual kinetic term, the topological phase occurs for $\mu>0$.

Because of Eq. (6), the spectrum is symmetric around $E=$ 0 . For $m=0$, there is a single solution with $l=0, E=0$ :

$$
u_{0}=v_{0} \sim \frac{1}{\sqrt{R(r)}} e^{-s \mu r / \Delta} .
$$

Note that this zero mode is present for any value of the angle $\alpha$, as long as $m \in 2 \mathbb{Z}$.

There is also a zero mode $\sim e^{-\mu\left(r_{o}-r\right) / \Delta}$ located at the other edge. Strictly speaking, these solutions are correct only for an infinitely extended cone, i.e., $r_{o} \rightarrow \infty$. However, they provide a very good approximation, as long as the distance between the edges is large compared to the size $\Delta / \mu$ of the zero mode since the overlap with the other edge will be exponentially small. In the limit $R_{i} \rightarrow \infty$, the expression for the full spectrum takes the simple form

$$
\begin{gathered}
u_{l}(r) \sim \exp \left[-\left(\frac{s \mu}{\Delta}+\frac{\cos \alpha}{2 R_{i}}\right) r\right], \\
E_{l}=\frac{\Delta}{R_{i}} l s,
\end{gathered}
$$

and again, we see that the system contains boundary modes if $\mu s>0$. Furthermore, the Majorana boundary mode at zero energy occurs only if also $l=0$, which can only happen when $m$ is even.

We now have all the information needed to answer one of the questions raised in the Introduction: what happens when we start from a cylinder, penetrated by an even number of flux quanta, and slowly squash it into an annulus by changing the angle $\alpha$ ? The zero mode from Eq. (16) remains as $\alpha$ is decreased towards zero, but the state will now carry current, as we can see from Eq. (12). By using the order parameter $\phi$ that minimizes Eq. (12), one sees that for $\alpha<\pi / 3$, the state with $n-m=1$ carries the smallest current, while for $\alpha>\pi / 3$ it is the $n-m=0$ state. Assuming that the energy is a monotonic function of the current, the ground states on the cylinder and the annulus are not adiabatically connected. As we have seen, for an integer number of flux quanta passing through the hole of the cone and $\alpha \neq 0, \pi / 2$, we necessarily are in a current carrying state, and at exactly $\alpha=\pi / 3$ there are two degenerate states which, however, differ in that they carry macroscopic currents with the same strength but in opposite directions. Furthermore, they differ in that only one of them supports two Majorana modes that extend around the cone [16]. Thus, in spite of not knowing the actual many-body spectrum, we can conclude that any local perturbation could, at most, open a gap that is exponentially small in the system size.

At $\alpha=\pi / 2$, which corresponds to the cylinder, the zerocurrent ground state hosts Majorana modes on the edges. Thus, if we make an adiabatic change of $\alpha$, the resulting state on the annulus will have a zero-energy mode, but it will not be the ground state. The ground state, on the other hand, will have odd $m$ and thus will not support a Majorana. However, if we assume that there is a magnetic field and we allow the system to relax to the ground state by a phase slip in $\phi$, the resulting state on the annulus will enclose an extra flux, and the zero-current state will then support a Majorana.

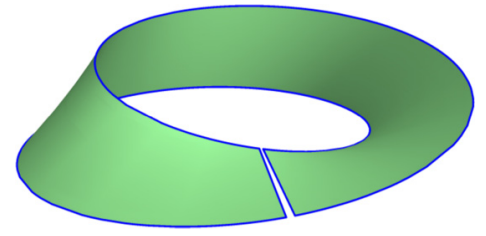

FIG. 2. A Möbius band is formed by gluing together the two edges of a rectangle with a twist. Because one twists the strip before gluing, the Möbius band is nonorientable and has only a single edge.

\section{EDGE MODES ON THE MÖBIUS BAND}

Through the example of a cone, we have shown how inducing a nonlocal geometric change to a surface can modify the properties of a $p$-wave superconductor. This occurs because the cone has a curvature defect in the hole, which vanishes for the annulus and reaches $2 \pi$ for the cylinder. However, it is not necessary to introduce curvature defects to create surfaces with nontrivial geometric effects. To further explore this possibility, we now turn to the Möbius band and use arguments based on the intrinsic geometry to determine the form of the ground state and the number and structure of the zero modes at the edge.

Considering only the intrinsic geometry, a flat Möbius band is a rectangle where the points of two opposite sides are identified via a reflection. If one considers an embedding in real three-dimensional (3D) space, one identifies the two edges after a twist (see Fig. 2).

Since the Möbius band is not orientable, there is no globally defined orthonormal frame $\left\{e_{a}\right\}_{a=1,2}$. Hence, it is impossible to have a uniform chiral superconductor on the Möbius band. However, for large enough systems, it is possible to induce such phases locally, from which we conclude that the system, by necessity, must exhibit defect lines. There are two topologically distinct choices for such a defect line, which are depicted in Fig. 3. These are minimal in the sense that you can generate all other domain wall configurations by either deforming these lines or by adding new lines surrounding contractible regions.

The configuration that first comes to mind is a defect along the line in Fig. 2, where the gap is closed by gluing. This results in a Möbius band with a transverse domain wall, as illustrated in Fig. 3(a). In order to perform a microscopic calculation

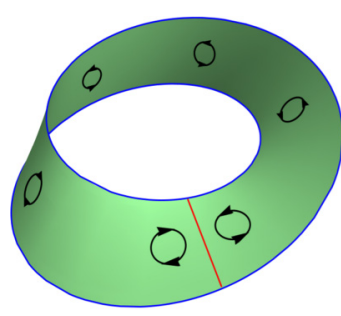

(a)

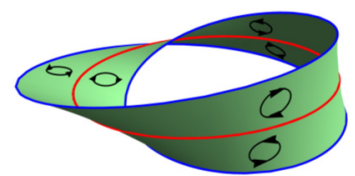

(b)
FIG. 3. Illustration of the two different types of line defects on a Möbius band supporting a chiral superconductor. The edge of the strip is shown in blue, and the line defect is shown in red. The circular arrows indicate the chirality of a $p$-wave superconducting order parameter that is constant along the strip, except at the defect. 


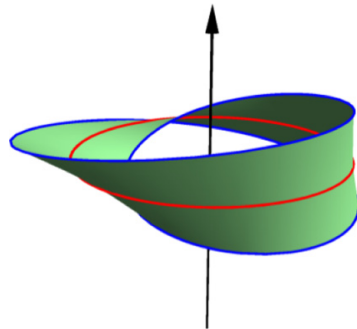

(a)

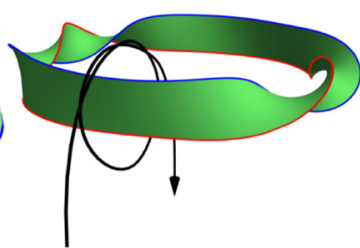

(b)
FIG. 4. (a) The Möbius band with a domain wall configuration (in red), as in Fig. 3(b). The arrow depicts a flux quantum threaded through the hole. (b) The Möbius band from (a), but cut along the domain wall. After this cut, the band turns into a cylinder with a $4 \pi$ twist, and the flux quantum winds around the band twice.

for this configuration, it is necessary to model the behavior of the order parameter at the end points of the defect, where two normal edge modes of opposite chirality meet to form a "double" mode along the domain wall. We shall not construct such a model here.

It is more interesting to consider a longitudinal line defect, which lies along the center line of the Möbius band, as shown in Fig. 3(b). By cutting the Möbius band along the defect line, one obtains a cylindrical geometry (the intrinsic geometry is insensitive to the $4 \pi$ twist), as depicted in Fig. 4. Using this, we can explain the presence of Majorana modes in the Möbius band by considering the results for the cylinder in the previous section. Imagine that the line defect is not just a line but is widened to form a thin gap, which we then further widen. Since our model only depends on the intrinsic geometry, there would now be no difference from the canonical cylindrical geometry. In this case, we know when the edge Majorana modes exist from the calculations in Sec. III. Since closing the gap will only affect the physics locally at one edge of the cylinder and since the Majorana modes occur and disappear in pairs, the edge Majorana will not disappear when the cylinder is glued back into a Möbius band. With no flux and for a large enough strip, we will thus have one zero-energy Majorana on the edge of the Möbius band and another at the domain wall.

To first order in the derivatives, the edge mode for the Möbius band will be given by the same solution as that for a cylinder, but to better describe the Majorana mode density profile at the line defect, we also need to take into account second-derivative terms in the Hamiltonian. For example, adding a quadratic derivative makes the Majorana wave function change under the addition of a superconducting flux quantum through the hole, even though it does not change the existence of the mode. To be concrete, take

$$
h_{0}=-\frac{\nabla^{2}}{2 M}-\mu
$$

in Eq. (3), where $M$ is the mass of a single Cooper pair. This choice will also explicitly demonstrate the statement made earlier, that higher-order terms select the boundary condition $s=1$ in Eq. (5). Thus, the requirement for the existence of the Majorana mode is, in fact, $\mu>0$.

To construct a coordinate system adapted to the domain wall, we let $x$ denote the length along the domain wall and $y$ denote the distance perpendicular to it. If the domain wall has length $L$, the twist in the Möbius band leads to the boundary condition $(L, y) \sim(0,-y)$. This coordinate system is natural in the sense that the domain wall is parametrized by the points $(x, 0)$. We will take $y \in \mathbb{R}$, so that the Möbius band is infinitely wide, which is equivalent to ignoring the exponentially small splitting that will occur between the Majorana particles due to finite-size effects.

Upon crossing the line defect, the chirality of the superconductor should be flipped, as shown in Fig. 3(b). For example, if $\phi_{+} \neq 0$ on one side of the line defect, then $\phi_{-} \neq 0$ on the other side. This is equivalent to changing $\partial_{+}$into $\partial_{-}$in Eq. (3) and vice versa when we cross the line defect. This procedure can be effected by replacing $\partial_{-}$with

$$
\tilde{\partial}_{-}:=e_{1}^{\mu} \partial_{\mu}-i \operatorname{sgn}(y) e_{2}^{\mu} \partial_{\mu},
$$

with a similar expression for $\partial_{+}$. Because the metric is Euclidean, the connection form vanishes, and we can make the gauge choice $\phi=\Delta$. We thus end up with the off-diagonal term [see Eq. (4)]

$$
\mathcal{H}_{12}=\frac{1}{2 \sqrt{g}}\left\{\sqrt{g} \phi, \tilde{\partial}_{-}\right\}=\Delta\left[\partial_{x}+i \operatorname{sgn}(y) \partial_{y}+i \delta(y)\right]
$$

in the first quantized Hamiltonian $\mathcal{H}$, which becomes

$$
\mathcal{H}=\left(-\frac{\nabla^{2}}{2 M}-\mu\right) \sigma^{z}+\Delta\left\{\sigma^{x} \partial_{x}+\sigma^{y}\left[\operatorname{sgn}(y) \partial_{y}+\delta(y)\right]\right\} .
$$

Next, we make the ansatz $\mathcal{H}(u, v)^{T}=0$ to find the solution of the Majorana equation

$$
\left(\begin{array}{c}
e^{-i \pi / 4} u(y) \\
e^{i \pi / 4} v(y)
\end{array}\right)=\left\{\begin{array}{cc}
\exp \left(-\alpha_{1}|y|\right) \chi & y>0, \\
\exp \left(-\alpha_{-1}|y|\right) \chi & y<0,
\end{array}\right.
$$

where $\chi$ is a constant column vector and $\alpha_{ \pm 1}$ are positive constants. After multiplying the Majorana equation on the left by $\operatorname{diag}\left(e^{-i \pi / 4}, e^{i \pi / 4}\right)$ and $\sigma_{z}$, we obtain the two equations (one for $\xi=1$ and one for $\xi=-1$ )

$$
0=\left\{-\frac{\alpha_{\xi}^{2}}{2 M}+\frac{\alpha_{1}+\alpha_{-1}}{2 M} \delta(y)-\mu+\Delta\left[\alpha_{\xi}-\delta(y)\right] \sigma_{y}\right\} \chi .
$$

Now, put $\chi=\chi_{\epsilon}$, where $\epsilon= \pm 1$ and $\sigma_{y} \chi_{\epsilon}=\epsilon \chi_{\epsilon}$. We then obtain

$$
\begin{aligned}
0 & =-\frac{\alpha_{\xi}^{2}}{2 M}-\mu+\epsilon \Delta \alpha_{\xi}, \\
\epsilon \Delta & =\frac{\alpha_{1}+\alpha_{-1}}{2 M} .
\end{aligned}
$$

From Eq. (24a), we find the four possible solutions (recall $\xi= \pm 1)$

$$
\alpha_{\xi}^{ \pm}=-M\left(-\epsilon \Delta \pm \sqrt{\Delta^{2}-2 \frac{\mu}{M}}\right),
$$

which are positive if and only if $\epsilon=1$ and $\mu>0$. These solutions are twofold degenerate since they do not depend on $\xi$. Then, Eq. (24b) is satisfied for the two parameter pairs $\left\{\alpha_{1}^{1}, \alpha_{-1}^{-1}\right\}$ and $\left\{\alpha_{-1}^{1}, \alpha_{1}^{-1}\right\}$ in Eq. (23). The solution on the Möbius band needs to satisfy $u(y)=u(-y)$ since it must obey 
$u(x+L, y)=u(x,-y)$ because of the boundary condition. Therefore, on the Möbius band without flux, we have a unique solution, which is the symmetric combination of the two solutions in Eq. (23),

$$
u(y)=-v(y) \propto\left[\exp \left(-\alpha_{1}^{1}|y|\right)+\exp \left(-\alpha_{1}^{-1}|y|\right)\right]
$$

where we used $\chi_{+} \propto\left(-e^{i \pi / 4}, e^{-i \pi / 4}\right)^{T}$.

It is now instructive to take the $M \rightarrow \infty$ limit to obtain the solution without the second-order derivative. In this limit, we find

$$
u(y)=-v(y) \propto \exp (-\mu|y| / \Delta),
$$

which is indeed the solution that you would obtain with boundary condition $s=1$ in Eq. (5).

\section{FLUXES AND VORTICES IN A $p$-WAVE MÖBIUS BAND}

We already described how the Möbius band in Fig. 2 can be obtained by twisting and gluing a rectangle. From practical experience with strips of paper, we expect that such a procedure could be done while keeping the geometry locally flat. Since paper does not stretch, any shape one can form out of paper without folding or cutting is intuitively isometric to the plane. Although their expressions are not simple, flat embeddings of the Möbius band into real 3D space have actually been found [17].

In a similar manner, the configuration in Fig. 3(b), which has a local chirality, can be formed from an oriented system. We know that cutting a Möbius band along the center line will produce a cylinder with a $2 \pi$ twist. Thus, by starting from a chiral state on such a twisted cylinder, we can fold it into a band, as in Fig. 3(b), but with a gap instead of a domain wall. One can then close the gap in a limiting procedure, obtaining a Möbius band with chirality as depicted in Fig. 3(b).

Let us now analyze what happens when a flux is threaded through the hole of the Möbius band. We can understand this configuration by viewing the Möbius band minus the line defect as an embedding of a cylinder, as depicted in Fig. 4(b). The winding number around the edge of a flux line passing through the hole in the Möbius strip is two rather than one, and the same holds for the winding number around the line defect. To better understand this statement for the line defect, consider that the latter consists, at each point, of two edge points glued together and that this doubling also doubles the winding number. Hence, whatever flux we thread through the Möbius band, it will correspond to twice that amount of flux through the cylinder, as shown in Fig. 4. Therefore, the magnetic flux through the hole of the Möbius band is not quantized in unit flux quanta but in half-unit flux quanta $[18,19]$. Because of this behavior, the Majoranas on the Möbius band can be created/destroyed by the addition of half a unit flux through the central hole.

In contrast, one can still only pierce a unit flux through the surface of the band, which will localize a Majorana at the vortex. This must, at the same time, add or remove a Majorana either on the edge or on the line defect. However, as illustrated in Fig. 5, no matter how the flux is pierced through, it will always, when the Möbius band is viewed as a cylinder, wind the edge corresponding to the line defect an even number of

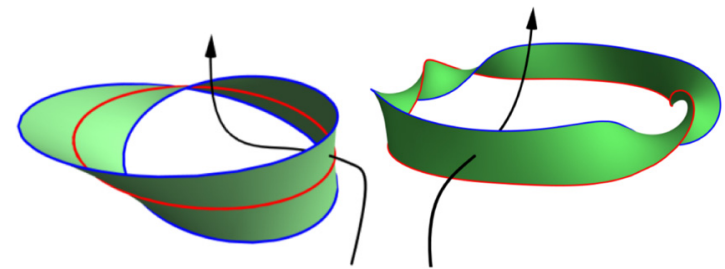

(a)

(b)

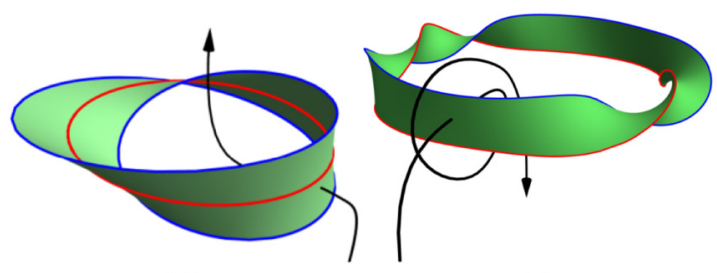

(c)

(d)

FIG. 5. (a) One of two inequivalent ways to thread a flux through the surface of the Möbius band. The flux line only encircles the edge and not the line defect. (b) The situation obtained by cutting the Möbius band from (a) along the line defect. It is explicitly visible that the flux line winds once around the edge and not around the line defect. (c) One of two inequivalent ways to thread a flux through the surface of the Möbius band. The flux line encircles both the edge and the line defect. (d) The situation obtained by cutting the Möbius band from (c) along the line defect. It is now clear that the flux line winds once around the edge and twice around the domain wall.

times and thus cannot alter the existence of a Majorana mode there.

As we show now, even though piercing a flux through the Möbius surface cannot destroy the Majorana at the line defect, adding the quadratic derivative term to the Hamiltonian allows one to distinguish different density profiles for the Majorana mode across the line defect. Adding a flux through the hole of the Möbius band corresponds to adding a vector potential $A_{x}=\pi /(e L)$ in the $(x, y)$ coordinate system constructed for the line defect. In the presence of this vector potential, $p_{x}$ can only vanish for a solution of the form $\exp (-i \pi x / L) \phi(y)$. To fulfill the condition $u(x+L, y)=u(x,-y)$, we need a solution antisymmetric in $y$, and we eventually obtain

$$
\begin{aligned}
u(y) & =-v(y) \\
& \propto e^{-i \pi x / L} \operatorname{sgn}(y)\left[\exp \left(-\alpha_{1}^{1}|y|\right)-\exp \left(-\alpha_{1}^{-1}|y|\right)\right],
\end{aligned}
$$

which, in the $M \rightarrow \infty$ limit, becomes

$$
u(y)=-v(y) \propto \operatorname{sgn}(y) \exp (-\mu|y| / \Delta) .
$$

Notice that for finite $M$ the density profile, e.g., $|u(y)|^{2}$, is different for the symmetric and the antisymmetric solutions, while for $M \rightarrow \infty$ it is not.

\section{CONCLUSIONS}

In the ground state, there are Majorana zero modes present at the edges for a cylindrical geometry but not for an annulus. We have shown that this apparent inconsistency can be understood by studying how a $p$-wave superconductor is affected by holonomies, i.e., curvature and curvature defects. By tackling this more general problem, we have also described what happens when one continuously deforms a cylinder 
into an annulus. Deforming the cylinder in this manner will result in an annulus with a supercurrent encircling the hole. This current carrying state will be degenerate due to the presence of Majorana modes, while the ground state will not be.

We have also studied the Möbius band, which can only host Majorana edge modes in the presence of defect lines. If one chooses a defect line along the center of the band, it is essentially a cylinder, with the edge corresponding to one side and the line defect to another. The novel behavior lies in the response to flux insertion; since the flux lines are mapped in a nontrivial way by the band-cylinder mapping, only specific flux configurations are allowed in the cylinder picture. A flux line through the hole of the band always winds twice in the cylinder picture, and hence, these fluxes are quantized at half integers. A flux line through the surface of the band is integer quantized and winds once around the edge but twice around the domain wall in the cylinder picture. Consequently, these fluxes do not remove the Majorana at the line defect, but they can change its density profile. Although the results discussed here are mostly of academic interest and 2D $p$-wave superconductors remain elusive [20], it might be possible to generate $p$-wave superfluids with fermionic ultracold atoms [21,22]. Moreover, Möbius bands might also become reality by exploiting a synthetic dimension generated by an internal degree of freedom such as spin in high-spin atoms and producing a twist via Raman-induced hopping at the edges of a ribbon, as suggested recently [23]. We hope that our results will stimulate experimental efforts in these directions.

\section{ACKNOWLEDGMENTS}

We thank Gi. Palumbo for helpful discussions and for comments on the manuscript. The work by A.Q. and C.M.S. is part of the D-ITP consortium, a program of the Netherlands Organisation for Scientific Research (NWO) that is funded by the Dutch Ministry of Education, Culture and Science (OCW). T.H.H. and T.K. were partially supported by the Swedish Research Council.
[1] G. Moore and N. Read, Nucl. Phys. B 360, 362 (1991).

[2] N. Read and E. Rezayi, Phys. Rev. B 54, 16864 (1996).

[3] N. Read and E. Rezayi, Phys. Rev. B 59, 8084 (1999).

[4] N. Read and D. Green, Phys. Rev. B 61, 10267 (2000).

[5] M. Greiter, X. Wen, and F. Wilczek, Nucl. Phys. B 374, 567 (1992).

[6] D. A. Ivanov, Phys. Rev. Lett. 86, 268 (2001).

[7] A. Stern, F. von Oppen, and E. Mariani, Phys. Rev. B 70, 205338 (2004).

[8] V. Mourik, K. Zuo, S. M. Frolov, S. R. Plissard, E. P. A. M. Bakkers, and L. P. Kouwenhoven, Science 336, 1003 (2012).

[9] H. O. H. Churchill, V. Fatemi, K. Grove-Rasmussen, M. T. Deng, P. Caroff, H. Q. Xu, and C. M. Marcus, Phys. Rev. B 87, 241401 (2013).

[10] Y. E. Kraus, A. Auerbach, H. A. Fertig, and S. H. Simon, Phys. Rev. B 79, 134515 (2009).

[11] S. Moroz, C. Hoyos, and L. Radzihovsky, Phys. Rev. B 93, 024521 (2016).

[12] W. Beugeling, A. Quelle, and C. Morais Smith, Phys. Rev. B 89, 235112 (2014).

[13] For any physical system the Hamiltonian would also have terms which does not depend on the intrinsic geometry, but for simplicity we do not consider such terms here.

[14] For the interaction to be well defined, the Fourier transform $V(q)$ of the potential $V(r)$, where $r$ is the geodesic distance, has to vanish as $q \rightarrow \infty$. However, the precise behavior at large momenta is not important for the long-wavelength physics.

[15] Adding higher-order terms would also mean that the ansatz $\Delta \phi=$ const is not a self-consistent solution close to vortices or edges.

[16] Remember that we use "Majorana" to refer to exact zero-energy modes only ("exact" meaning exponentially small in the system size). It is only the presence of these zero-energy modes that distinguishes the two states; they are both describing the same topological phase of matter.

[17] There are many such flat embeddings, and it is interesting to ask which one would minimize the elastic energy for a real physical Möbius band. This problem was recently solved numerically in Ref. [24].

[18] M. Hayashi and H. Ebisawa, J. Phys. Soc. Jpn. 70, 3495 (2001).

[19] M. Hayashi, H. Ebisawa, and K. Kuboki, Phys. Rev. B 72, 024505 (2005).

[20] Y. Maeno, S. Kittaka, T. Nomura, S. Yonezawa, and K. Ishida, J. Phys. Soc. Jpn. 81, 011009 (2012).

[21] N. R. Cooper and G. V. Shlyapnikov, Phys. Rev. Lett. 103, 155302 (2009).

[22] Y. Nishida, Ann. Phys. (N.Y.) 324, 897 (2009).

[23] O. Boada, A. Celi, J. Rodríguez-Laguna, J. I. Latorre, and M. Lewenstein, New J. Phys. 17, 045007 (2015).

[24] E. L. Starostin and G. H. M. van der Heijden, Nat. Mater. 6, 563 (2007). 